\title{
Level density within a micro-macroscopic approach
}

\author{
A.G. Magner ${ }^{\text {a,* }}$, A.I. Sanzhur ${ }^{\mathrm{a}}$, S.N. Fedotkin ${ }^{\mathrm{a}}$, A.I. Levon ${ }^{\mathrm{a}}$, S. Shlomo ${ }^{\mathrm{b}}$ \\ ${ }^{a}$ Institute for Nuclear Research, 03028 Kyiv, Ukraine \\ ${ }^{b}$ Cyclotron Institute, Texas AEM University, College Station, Texas 77843, USA
}

\begin{abstract}
Statistical level density $\rho(E, A)$ is derived for nucleonic system with a given energy $E$, particle number $A$ and other integrals of motion in the micro-macroscopic approximation beyond the standard saddle-point method of the Fermi gas model. This level density reaches the two limits; the well-known Fermi gas grand-canonical ensemble limit for a large entropy $S$ related to large excitation energies, and the finite micro-canonical limit for a small combinatorical entropy $S$ at low excitation energies. The inverse level density parameter $K$ as function of the particle number $A$ in the semiclassical periodic orbit theory, taking into account the extended Thomas-Fermi and Strutinsky shell corrections, is calculated and compared with experimental data.
\end{abstract}

Keywords: level density, shell effects, Thomas-Fermi approach, periodic orbit theory, neutron resonances, arXiv:2006.03868v3 [nucl-th].

\section{Introduction}

Many properties of heavy nuclei can be to large extent described in terms of the statistical level density [1-24]. Usually, the level density $\rho(E, A)$, where $E$ and $A$, are the energy and nucleon number, respectively, is calculated by the inverse Laplace transformation of the partition function $\mathcal{Z}(\beta, \alpha)$ of the corresponding Lagrange multipliers. Other integrals of motion, e.g., the orbital angular momentum projection $M$, or separately, the number of neutrons and protons instead of $A$ can be considered in a similar way by introducing other Lagrange multipliers. Within the grand canonical ensemble, the standard saddle-point method (SPM) was used for the integration over all variables, including $\beta$, which is related to the total energy $E[2,4]$. This method assumes a large excitation energy $U$, so that the temperature $T$ can be defined through a well-determined saddle point in the integration variable $\beta$ for a finite Fermi system of large particle numbers. However, many experimental data are also related to the low-lying part of the excitation energy $U$, where such a saddle point does not exist. For presentation of experimental data on nuclear spectra, the cumulative number of quantum levels below a given excitation energy $U$ is conveniently often used for a statistical analysis [25-27], e.g., of the experimental data on the collective excitation energies of rare-earth and actinide nuclei excited in two-neutron transfer $(p, t)$ reactions [28]. For calculations of this cumulative number one has to integrate the level density over a large interval of the excitation energy $U$ from small values where there is no thermodynamical equilibrium to large values where the standard approach can be successfully applied in terms of the temperature $T$ in a finite Fermi system. Therefore, to simplify the level density, $\rho(E, A)$, calculations we will integrate over the Lagrange multiplier $\beta$ in the inverse Laplace transformation of the partition function $\mathcal{Z}(\beta, \alpha)$ more accurately beyond the SPM. It is worthwhile to unify the standard Fermi-gas model for large excitation energies $U$ with the empiric constant temperature model at small $U$ as suggested in Ref. [3], see also Refs. [17, 20, 21].

More general microscopic formulation of the energy level density for mesoscopic systems, in particular for nuclei, which removes the singularity at small excitation energies, is discussed in Ref. [21], see also references therein. One of the microscopic ways for accounting for interparticle interactions beyond the mean field (shell model) in the level density calculations was suggested within the Monte-Carlo Shell Model [12, 15, 29]. Another successful approach

\footnotetext{
${ }^{*}$ Corresponding author

Email address: magner@kinr.kiev. ua (A.G. Magner)
} 
for taking into account the interparticle interactions above the simple shell model is given by the moments method $[18,20,23,25,30]$. The main ideas are based on the random matrix theory, see Refs. [25, 30-32].

A micro-macroscopic approximation (MMA) which unifies micro- and macroscopic ensembles, for the statistical level density $\rho$ was suggested in Ref. [33]. This MMA approach is based on the Strutinsky shell correction method [34-36] within the Landau-Migdal quasiparticle theory called as the Finite Fermi System Theory [37, 38]. A mean field potential is used for calculations of the energy shell corrections, $\delta E$. The total nuclear energy, $E$, is the sum of these corrections and smooth macroscopic liquid-drop component [39] which can be well approximated by the Extended Thomas-Fermi ((E)TF) approach $[40,41]$. Thus, within the semiclassical approximation to the Strutinsky shell correction method, the interactions between particles, in particular interparticle collision-like interactions, averaged over particle numbers, i.e. over many-body microscopic quantum states in realistic nuclei, are approximately taken into account through the extended Thomas-Fermi component beyond the mean field. In this way, one can present [33] the level density $\rho$ in terms of the modified Bessel function of the entropy variable in the case of small thermal excitation energy $U$ as compared to the rotational energy $E_{\text {rot }}$. The shell-correction method [34, 35] was applied [33] for studying the shell effects in the nuclear moment of inertia. For a deeper understanding of the correspondence between the classical and the quantum approach, it is also worthwhile to analyze the shell effects in the level density $\rho$ within the semiclassical periodic-orbit theory (POT) [41-45].

In the present study we extend, in a simple transparent way, the MMA approach [33] for the description of shell effects in terms of the level density itself from small to large excitation energies $U$, see also detailed derivations accounting for the rotational and isotopically asymmetric effects in Refs. [46, 47]. The level density parameter $a$ is one of the key quantities under intensive experimental and theoretical discussions [7, 9, 13, 17, 19, 24]. Properties of the smooth inverse level density parameter, $K=A / a$, as function of the nucleon number $A$ have been studied within the framework of the self-consistent ETF approach [9, 19]. However, shell effects in the statistical level density is still an attractive subject, especially important near nuclear magic numbers.

The structure of the paper is the following. In Sec. 2, the level density $\rho(E, A)$ is derived within the MMA for the one-component nucleon system by using the POT. We discuss general properties of the statistical level density $\rho(S)$ and its approximations as function of the entropy $S$. In Section 3, we compare our analytical MMA results for the level density $\rho(E, A)$, and the inverse level density parameter $K$ with experimental data for several nuclei as typical examples. Our Conclusions are presented in Section 4. Some details of the periodic orbit theory are presented in Appendix A.

\section{Micro-macroscopic approach}

For a statistical description of level density of a nucleus in terms of the conservation variables - the total energy, $E$, and nucleon number, $A$, one can begin with the microcanonical expression for the level density,

$$
\rho(E, A)=\sum_{i} \delta\left(E-E_{i}\right) \delta\left(A-A_{i}\right) \equiv \int \frac{\mathrm{d} \beta \mathrm{d} \alpha}{(2 \pi i)^{2}} \exp [S(\beta, \alpha)],
$$

where $E_{i}$ and $A_{i}$ present the system spectrum, $S(\beta, \alpha)=\ln \mathcal{Z}(\beta, \alpha)+\beta E-\alpha A$ is the entropy with $\mathcal{Z}(\beta, \alpha)$ being the partition function. Integrating over $\alpha$ for a given $\beta$ by the standard SPM in Eq. (1), we use the expansion for this entropy as: $S(\beta, \alpha)=S\left(\beta, \alpha^{*}\right)+\left(\partial^{2} S / \partial \alpha^{2}\right)^{*}\left(\alpha-\alpha^{*}\right)^{2} / 2+\ldots$. The first order term of this expansion disappears because the Lagrange multiplier, $\alpha^{*}$, is defined by the saddle point condition,

$$
\left(\frac{\partial S}{\partial \alpha}\right)^{*} \equiv\left(\frac{\partial \ln \mathcal{Z}}{\partial \alpha}\right)^{*}-A=0
$$

Introducing, for convenience, the potential $\Omega=-\ln \mathcal{Z} / \beta$, one can define the system partition function $\mathcal{Z}$ through

$$
\Omega=\Omega_{0}-\frac{a}{\beta^{2}}, \quad \Omega_{0}=E_{0}-\lambda A,
$$

where $E_{0}$ is the energy of a non-excited ground-state system, $a$ is the level density parameter, and $\lambda=\alpha^{*} / \beta$ is associated with the chemical potential as function of the particle number $A$ at arbitrary $\beta$ through Eq. (2). Substituting 
the expansion of the entropy near the saddle point into Eq. (1), and taking the error integral over $\alpha$ in the infinite limits, one obtains

$$
\rho(E, A) \approx \int \frac{\mathrm{d} \beta \beta^{1 / 2}}{2 \pi i \sqrt{2 \pi}} \mathcal{J}^{-1 / 2} \exp \left(\beta U+\frac{a}{\beta}\right),
$$

where $U=E-E_{0}$ is the excitation energy. In the thermodynamic limit for a large excitation energy $U$ one finds the temperature $T=1 / \beta^{*}$ through the saddle point $\beta=\beta^{*}$, and the well-known expression for the grand-canonical potential $\Omega, \Omega^{*}=\Omega_{0}-a T^{2}=\Omega_{0}-U$. In this limit, $\lambda=\alpha^{*} T$ is the usual chemical potential at the thermodynamic equilibrium with temperature $T$. In Eq. (4), the one-dimensional Jacobian determinant $\mathcal{T}$ ( $c$ number) is taken at the saddle point (2), i.e.,

$$
\mathcal{J} \equiv \beta\left(\frac{\partial^{2} S}{\partial \alpha^{2}}\right)^{*} \equiv-\left(\frac{\partial^{2} \Omega}{\partial \lambda^{2}}\right)^{*}=\mathcal{J}_{1}+\mathcal{J}_{2}, \quad \mathcal{J}_{1}=-\frac{\partial^{2} \Omega_{0}}{\partial \lambda^{2}}, \quad \mathcal{J}_{2}=\frac{1}{\beta^{2}} \frac{\partial^{2} a}{\partial \lambda^{2}}
$$

The level density parameter $a$ can be expressed in terms of the ETF level density and shell correction,

$$
a=\frac{\pi^{2}}{6} g(\lambda), \quad g(\lambda)=\tilde{g}(\lambda)+\delta g(\lambda),
$$

where $\tilde{g}(\varepsilon)$ is the Strutinsky smooth level density, approximately equal to the ETF one (including the spin or spinisospin degeneracy). The slightly averaged oscillating (shell) component $\delta g(\varepsilon)$ of the level density $g(\lambda)$ can be calculated using the shell-correction method [35] or approximately, within the semiclassical periodic orbit theory, see Appendix A and Refs. [41, 43]. Thus, for the level density parameter $a$ one has a decomposition of the Strutinsky shell correction method [34, 35]:

$$
a=\tilde{a}+\delta a, \quad \tilde{a}=\frac{\pi^{2}}{6} \tilde{g}, \quad \delta a=\frac{\pi^{2}}{6} \delta g,
$$

where $\tilde{a}$ is the smooth ETF component of the level density parameter $a, \tilde{a}=a_{\mathrm{ETF}}$, and $\delta a$ is its shell correction. Both components of the level density $g(\lambda)$ are taken at the chemical potential $\varepsilon \approx \lambda$. The shell correction part is averaged with Gaussian having the average parameter $\gamma$ which is much smaller than the distance between major shells. Using the mean field expressions for the energy $E_{0}$ and particle number $A$ in terms of the level density $g(\varepsilon)$, one has

$$
E_{0}=\int_{0}^{\lambda} \mathrm{d} \varepsilon \varepsilon g(\varepsilon), \quad A=\int_{0}^{\lambda} \mathrm{d} \varepsilon g(\varepsilon) .
$$

For the Jacobian component $\mathcal{J}_{1}$, one arrives at the approximation of smooth ETF level density, $g \approx \tilde{g}$, neglecting its derivatives contribution [4], $\mathcal{J} \cong \mathcal{J}_{1} \approx g(\lambda)$. This approximation is named below as the case (i). According to Eq. (6), for the case (ii) of the dominating second term (5) of the Jacobian $\mathcal{J}$ one writes

$$
\mathcal{J} \approx \mathcal{J}_{2} \approx\left(\pi^{2} / 6\right) g^{\prime \prime}(\lambda) / \beta^{2} \approx\left(\pi^{2} / 6\right) \delta g^{\prime \prime}(\lambda) / \beta^{2}
$$

see Eq. (A.13) for $\delta g^{\prime \prime}(\lambda)$. For following derivations it is helpful to introduce the Jacobian ratio ${ }^{1}$ given by

$$
\xi=\mathcal{J}_{2} / \mathcal{J}_{1} \approx\left(\pi^{2} / 6\right) \delta g^{\prime \prime}(\lambda) / \beta^{2} \tilde{g}(\lambda) \sim\left(4 \pi^{6} / 3\right)(T / \lambda)^{2} A^{1 / 3} \mathcal{E}_{\mathrm{sh}}, \quad \mathcal{E}_{\mathrm{sh}}=-\delta E A / E_{\mathrm{ETF}},
$$

where $T=1 / \beta^{*}=\sqrt{U / a}$ is the temperature evaluated through the saddle point $\beta=\beta^{*}, \delta E \approx \delta E_{\mathrm{scl}}$ is the semiclassical POT energy shell correction, Eq. (A.12), for major shell structure [41, 43-45], and $E_{\mathrm{ETF}}$ is the smooth ETF energy, $E_{\mathrm{ETF}} \sim \tilde{g}(\lambda) \lambda^{2}$. The derivatives $\delta g^{\prime \prime}$ are given in Eq. (A.13). For typical values of parameters $\lambda=40 \mathrm{MeV}, A \sim 200$, and relative energy shell correction $\mathcal{E}_{s h} \sim 0.2-2.0$ [48], one finds the estimate $\xi \sim 1-10$ for temperature $T \sim 1 \mathrm{MeV}$, much smaller than $\lambda$, and $\xi \sim 0.01-0.1$ for very small temperature $T \sim 0.1 \mathrm{MeV}$. In line with the shell correction method [35] and extended Thomas-Fermi approach [41], these values are given finally using the realistic smooth energy $E_{\mathrm{ETF}}$ for which the binding energy approximately equals $E_{\mathrm{ETF}}+\delta E$.

\footnotetext{
${ }^{1}$ We shall present the main case of $\delta E<0$ near the minimum of shell correction energy, as mainly applied below. For the case of a positive $\delta E$ we change, for convenience, signs so that we will get $\xi>0$.
} 

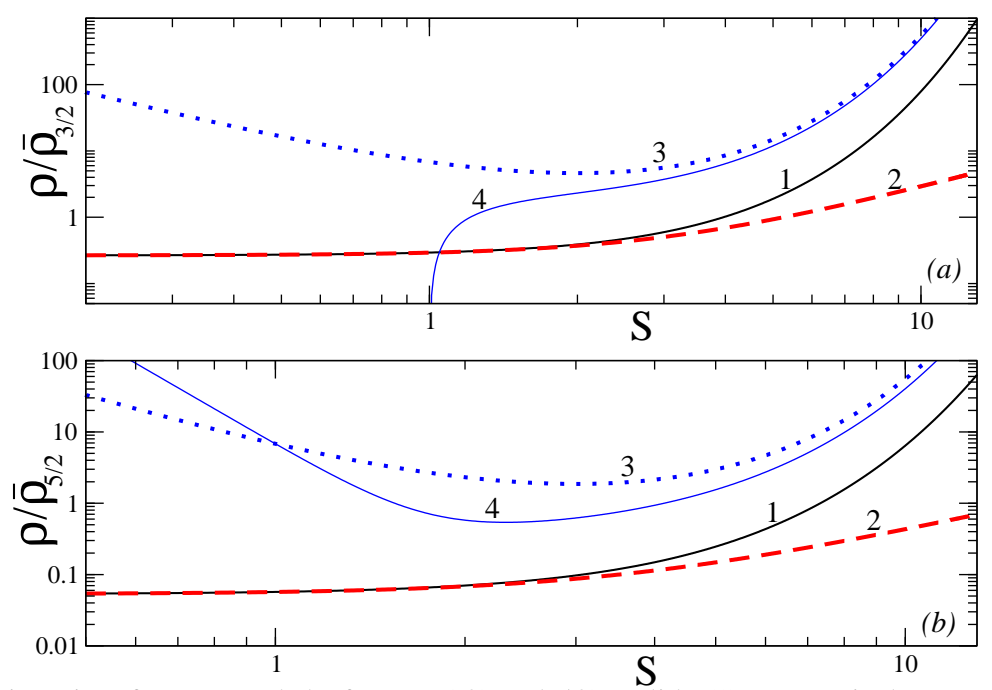

Figure 1: Level density $\rho$ in units of $\bar{\rho}_{v}$, (a) and (b) for $v=3 / 2$ and 5/2 (solid "1"), respectively, are shown as functions of the entropy $S$ in different approximations: 1) $S \ll 1$ (dashed "2") Eq. (17), and 2) $S \gg 1$ (dots and thin solid), Eq. (16), "3" and "4", are expansions over $1 / S$ up to zero and first [in (a)] or second [in (b)] order terms in Eq. (16), respectively.

For calculations of the Jacobian $\mathcal{J}$, Eq. (5), we will consider two different limiting cases (i) and (ii) (see above and more details in Refs. [46, 47]). In the case (i) of small contribution of the $\beta$-dependent component $\mathcal{J}_{2}$ of the Jacobian (5), $\xi \ll 1$, Eq. (10), that is related, for instance, to small heat excitations with respect to the collective (rotational) motion, we will approximate the Jacobian $\mathcal{J}$ by the constant $\mathcal{J}_{1}$ (independent of $\beta$ ), $\mathcal{J} \approx \mathcal{J}_{1}$. Then, one takes the Jacobian factor $\mathcal{J}^{-1 / 2}$ off the integral in Eq. (4), done as in Ref. [33]. Transforming $\beta$ to a new variable $\tau=1 / \beta$, we recognize in Eq. (4) the standard Laplace transform. Evaluating this integral over $\tau$, one finally arrives at

$$
\rho \approx \rho_{\text {MMA }}(S)=\bar{\rho}_{v} f_{v}(S), \quad f_{v}(S)=S^{-v} I_{v}(S),
$$

where $S$ is the entropy, $S=2 \sqrt{a U}$ with $U$ being the excitation energy defined above, $U=E-E_{0}$. This entropy $S$ can be expressed in terms of the level density parameter $a$ and excitation energy $U$ as in the mean field approach. However, the level density parameter $a$ [Eq. (7)] in our MMA approach contains the ETF part $\tilde{a}$ related to the liquid drop energy through the ETF level density $\tilde{g}$. In Eq. (11), $\bar{\rho}_{v}$ is a constant, independent of the entropy $S, \bar{\rho}_{v}=2 a^{v} \pi^{1-v}\left|\mathcal{T}_{1}^{(2 v-2)}\right|^{-1 / 2}$, where $2 v-2$ is the determinant dimension [this superscript is omitted in Eqs. (4) and (5)]. The modified Bessel function $I_{v}(S)$ of the order of $v, v=(n+1) / 2$, is determined by the number of integrals of motion $n(n=2$, and therefore, $v=3 / 2$ for this case of the two integrals of motion $E$ and $A$ ). Expression (11) is written in a general form for arbitrary number of integrals of motion $n$. For the specific case $n=2$ here $(v=3 / 2)$, within the same case (i) for relatively small shell-corrections contributions, named below also as the MMA1 approximation, one obtains

$$
\rho_{\text {MMA } 1}(E, A)=a \sqrt{2 \pi / 3} I_{3 / 2}(S) / S^{3 / 2}, \quad(i) ;
$$

see also Ref. [33] for the case of $n=3(v=2, \rho=\rho(E, A, M))$ in Eq. (11).

In the opposite case (ii) of the dominating shell-corrections contributions, $\xi \gg 1$, see Eq. (10), assuming a relatively large (heat) excitation energy with respect to the collective rotational energy, one obtains Eq. (9) for the Jacobian. The inverse Laplace integrand requires the additional power of $\beta$. Therefore, after transformation of the variable, $\beta=1 / \tau$, and taking explicitly the inverse Laplace transform we arrive at the same expressions of Eq. (11) but with $v=5 / 2$ (MMA2 approximation),

$$
\rho_{\mathrm{MMA}}(E, A)=\bar{\rho}_{5 / 2} S^{-5 / 2} I_{5 / 2}(S), \quad \bar{\rho}_{5 / 2}=4 a^{2}(\pi / 6 \bar{\xi})^{1 / 2}, \quad(i i) ;
$$

where

$$
\bar{\xi}=\beta^{2} \xi \approx \frac{4 \pi^{6} A^{1 / 3} \mathcal{E}_{\mathrm{sh}}}{3 \lambda^{2}},
$$


see Eq. (10) for $\mathcal{E}_{\text {sh }}$.

We will specify MMA2 approach (ii), Eq. (13), as MMA2a one for $\mathcal{E}_{\mathrm{sh}}$ taken in Eq. (14) from Ref. [48]. For the TF approximation to the coefficient $\bar{\rho}_{5 / 2}$ within the same case (ii) one finds (MMA2b approximation) [46]

$$
\rho_{\text {MMA } 2 \mathrm{~b}}(S)=\bar{\rho}_{5 / 2}^{(2 b)} S^{-5 / 2} I_{5 / 2}(S), \quad \bar{\rho}_{5 / 2}^{(2 b)} \approx 2 \sqrt{2 / \pi} \lambda a^{2}, \quad(i i) .
$$

For derivations of the coefficient, $\bar{\rho}_{5 / 2}^{(2 b)}$, we assumed in Eq. (13) for $\bar{\rho}_{5 / 2}$ that the magnitude of the relative shell corrections $\mathcal{E}_{\mathrm{sh}}, \bar{\xi} \propto \mathcal{E}_{\mathrm{sh}}$; see Eq. (14), is extremely small but their derivatives yield large contributions through the level density derivatives $g^{\prime \prime}(\lambda)$ [Eqs. (A.13)], $g \propto A / \lambda$, as in the TF approach. In this case for the spinprojection-dependent level density $\rho(E, A, M)$, one obtains the same expressions of Eq. (11) but with $v=3$ and $\bar{\rho}_{3} \approx\left(4 \sqrt{2} a^{3} \hbar / \pi^{2}\right)\left(\lambda^{3} / A \Theta\right)^{1 / 2}$ in Eq. (11), where $\Theta$ is the moment of inertia, which is approximated by the TF nuclear moment of inertia $\Theta_{\mathrm{TF}}, \Theta \approx \Theta_{\mathrm{TF}}$, see Ref. [46]. The values of $v$ in the case (ii) correspond to $v-1$ of the case (i). Subscript $v$ of the Bessel functions in Eq. (11) is increased by $1 / 2$ for isotopically asymmetric neutron-proton systems.

The asymptote for large entropy $S$ is given by

$$
f_{v}(S)=\frac{\exp (S)}{S^{v} \sqrt{2 \pi S}}\left[1+\frac{1-4 v^{2}}{8 S}+\mathrm{O}\left(\frac{1}{S^{2}}\right)\right] .
$$

This approximation at zero order in expansion over $1 / S$ is identical to that obtained directly from Eq. (4) by the SPM over all variables. At small entropy, $S \ll 1$, one obtains also from Eq. (11) the finite combinatoric power expansion $[2,49,50]:$

$$
f_{v}(S)=\frac{2^{-v}}{\Gamma(v+1)}\left[1+\frac{S^{2}}{4(v+1)}+\mathrm{O}\left(S^{4}\right)\right],
$$

where $\Gamma(x)$ is the gamma function. This expansion over powers of $S^{2} \propto U$ is similar to that of the "constant temperature model" $[3,17,20,21]$, used often for the level density calculations at small excitation energies $U$, but here we have it without free fitting parameters. Moreover, we do not need to match asymptotes for large, Eq. (16), and small, Eq. (17), excitation energies $U$ because they are connected analytically by the Bessel function, Eq. (11), and therefore, have no extra free fitting parameters for their matching in the MMA. Using the asymptotes of Eq. (16) in the case (i), Eq. (12), for large $S$, one arrives at the Bethe expression for the level density [1, 2, 4],

$$
\rho_{\mathrm{FG}}(E, A)=\frac{\exp (S)}{\sqrt{48} U}
$$

This asymptotic expression is obviously divergent at $U \rightarrow 0$, in contrast to the finite MMA limit (17) for the level density, Eq. (11).

Notice that the MMA1 approximation for the level density, $\rho(E, A)$, Eq. (12), can be applied also for large excitation energies, $U$, with respect to the collective rotational excitations, as the FG approximation if one can neglect shell effects, $\xi \ll 1$. Thus, the level density $\rho(E, A)$ in the case (i), Eq. (12), has a wider range of the applicability over the excitation energy variable $U$ than the MMA2 case (ii). The MMA2 approach has, however, another advantage of describing the important shell structure effects. We emphasize also that the main effects of the interparticle interaction, statistically averaged over particle numbers, beyond the shell correction of the mean field within the Strutinsky's shell correction method, was taken into account by the extended Thomas-Fermi components of MMA expression (11) for the level density, $\rho(E, A)$. These components are given by the extended Thomas-Fermi potential, $\Omega_{\mathrm{ETF}}$, Eq. (A.6), and the level-density parameter, $a_{\mathrm{ETF}}$, counterparts of the corresponding total quantities, Eqs. (A.4) and (7).

\section{Results and discussions}

In Fig. 1 we show the level density dependence $\rho(S)$ [Eq. (11), for $v=3 / 2$ in Fig. 1 (a) and $v=5 / 2$ in Fig. 1 (b) panels] on the entropy variable $S$ and its different asymptotes. In this figure, the results of a small $[S \ll 1$, Eq. (17)] and large $[S \gg 1$, Eq. (16)] entropy $S$ behavior are presented. For large $S \gg 1$ we neglected the corrections of the inverse power expansion of the pre-exponent factor in square brackets of Eq. (16), lines "3". We also took into 


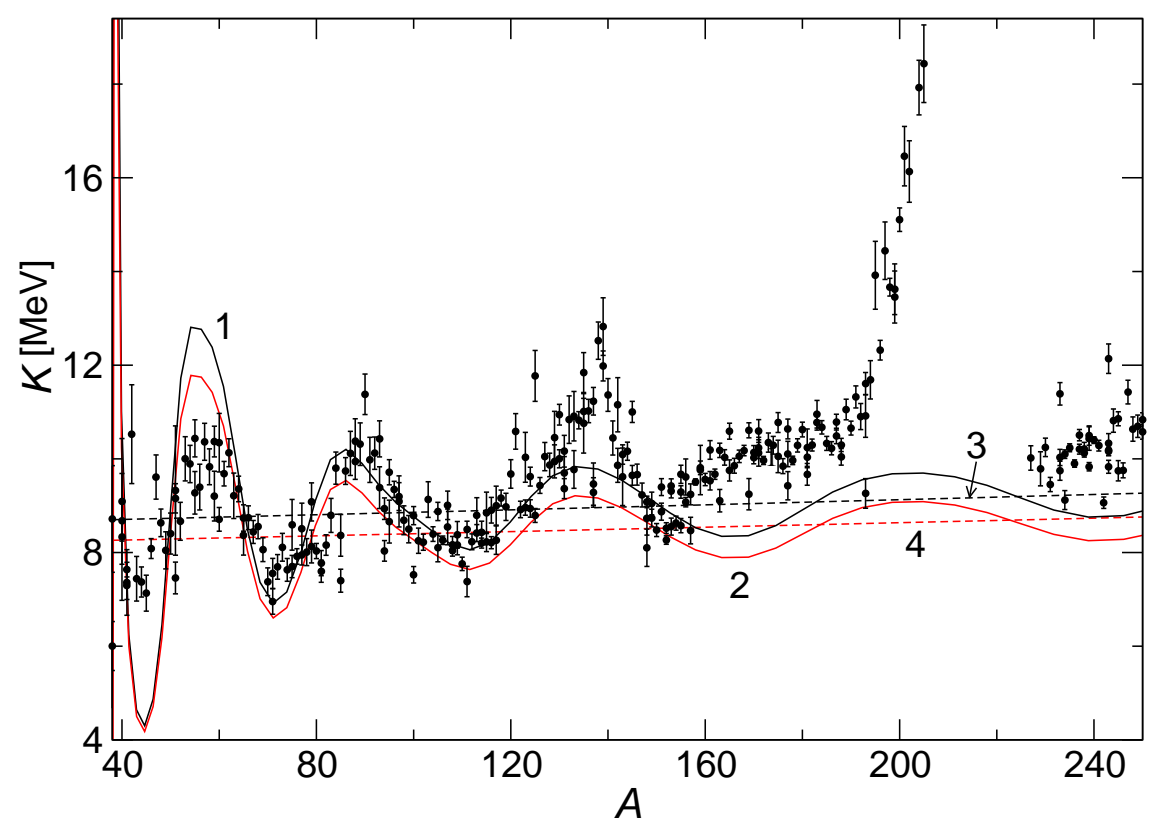

Figure 2: The inverse level-density parameter $K=A / a$ (solids "1" for SKM* and "2" for KDE0v1 forces) is shown as function of the particle number $A$. The smooth part in the ETF approach is taken from Ref. [19] for these two versions of the Skyrme forces SKM* ("3" dashed) and KDE0v1 ("4" dashed). The solid oscillating curves are obtained by using the semiclassical POT approximation, Eq. (A.2), for the level density shell corrections, Eq. (A.3), at Gauss width averaging parameter $\gamma=0.3$, and dashed curves "3" and "4" for a smooth part, both including the effective mass contribution. Experimental values, shown by solid points, are taken from Ref. [13].

account the corrections of the first $[v=3 / 2$, (a) $]$ and up to second $[v=5 / 2$, (b)] order in $1 / S$ to show their slow convergence to the exact MMA result "1" [Eq. (11)]. It is interesting to find almost a parallel constant shift of the simplest, $\rho \propto \exp (S) / S^{v}$, SPM asymptotic approximation at large $S$ (dots "3") with respect to the solid curve of the exact MMA result (11). This may clarify one of the phenomenological models, e.g., the back-shifted Fermi-gas (BSFG) model for the level density [8, 13, 51].

Fig. 2 shows the inverse level-density parameter, $K=A / a$, with $a$ of Eq. (6) as function of the particle number $A$ in the semiclassical POT approximation (see Appendix A). The result of these calculations are largely in a qualitative agreement with the recent experimental data [13], which, as compared to Ref. [5], included in the analysis of many other excited nuclei and different reactions with nuclear excitation energies being significantly smaller than the neutron separation energy. The sets with reliable completeness of levels in the limited energy range below the neutron binding energy were selected for each nucleus, and neutron resonance level densities were included in the analysis. We added the smooth self-consistent ETF values $\tilde{a}$ of $a$ [Eq. (7)] for the KDE0v1 [52] and the SkM* [40] Skyrme forces from Refs. $[19,24]$ to their shell corrections, $\delta g(\lambda)$, through the total level density $g(\lambda)$ of Eq. (6). Its oscillating component $\delta g(\lambda)$ was approximated by the analytical POT trace formula [41-43] for the infinitely deep spherical square-well potential; see Eqs. (A.3) and (A.2). This formula reproduces, almost identically, the quantum results of the shell correction method for the level density $g$ in the same potential [41]. The major closed shell nuclei are clearly recognized in Fig. 2 by the maxima of $K(A)$, which correspond to minima of the level density parameter $a$, or the oscillating level density component $\delta g(\lambda)$, see Eqs. (6) and (A.2). The relationship between the chemical potential $\lambda$, through $k_{F} R$, where $\hbar k_{F}=(2 m \lambda)^{1 / 2} \approx\left(2 m \varepsilon_{F}\right)^{1 / 2}$ is the Fermi momentum, $\varepsilon_{F}$ is the Fermi energy, and $m$ the nucleon mass. The particle numbers $A$ for this potential [41, 43] is given by the second equation of Eq. (8). In the transformation of $k_{F} R$ (or $\lambda$ ) to $A$, Eq. (8), one can conveniently use the quantum shell-correction level density [35]. The Gaussian averaging width of the oscillating level density $\delta g$ in Fig. 2 [see Eq. (A.3)] is the same, $\gamma=0.3$, as that adopted in previous POT calculations [45]. It corresponds to the dimensional Gaussian width $\Gamma \approx 2 \gamma \lambda / k_{F} R \approx 3$ $\mathrm{MeV}\left(\lambda=40 \mathrm{MeV}, R=r_{0} A^{1 / 3}, r_{0}=1.14 \mathrm{fm}\right.$, and $\left.A=100-200\right)$. In calculations results presented in Fig. 2 , only short planar POs yield the main major-shell contributions into the PO sum, Eqs. (A.3) and (A.2), for the oscillating 

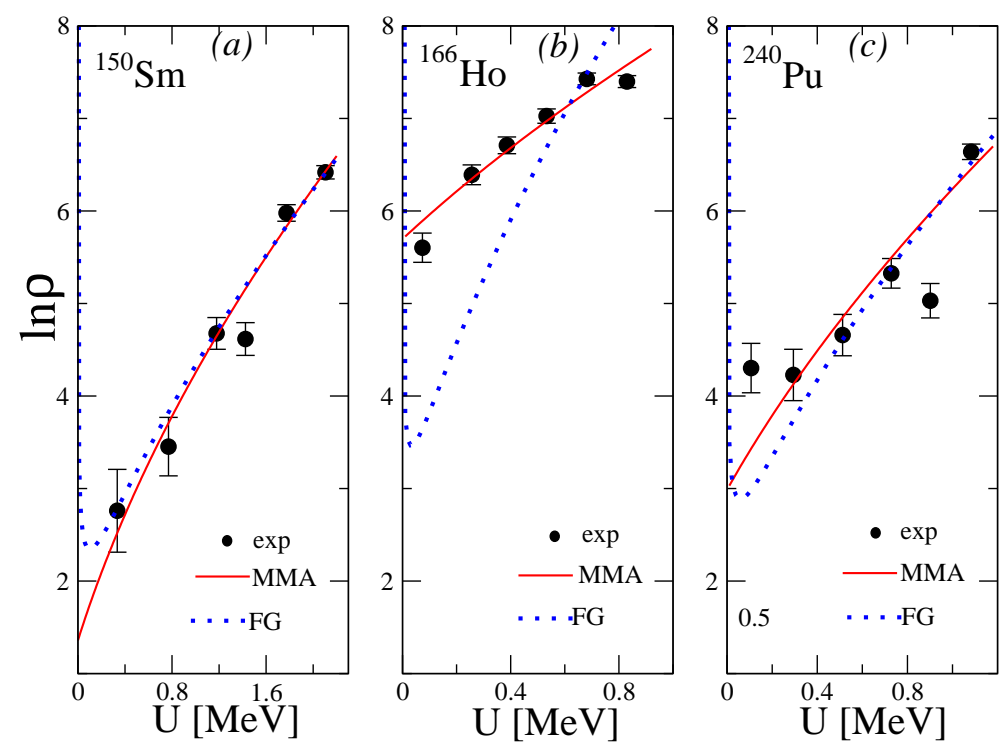

Figure 3: Level density, $\ln \rho(E, A)$, obtained for low energy states in nuclei ${ }^{150} \mathrm{Sm}(\mathrm{a}),{ }^{166} \mathrm{Ho}$ (b), and ${ }^{240} \mathrm{Pu}$ (c) in the MMA for the smallest $\sigma$, Eq. (19), in each nucleus among all MMA approximations, MMA1 [Eq. (12)], MMA2b [Eq. (15)], and MMA2a [Eq. (13)] with the realistic relative shell-energy corrections, $\mathcal{E}_{\text {sh }}$ from Ref. [48]), respectively; the chemical potential is constant, $\lambda=40 \mathrm{MeV}$, independent of particle numbers. The MMA is compared with the standard Fermi gas formula, Eq. (18). The relative shell corrections, $\mathcal{E}_{\mathrm{sh}}$, are taken from Ref. [48], $\mathcal{E}_{\mathrm{sh}}=0.2,0.5$ and 0.7 for the ${ }^{150} \mathrm{Sm}(\mathrm{a}),{ }^{166} \mathrm{Ho}$ (b) and ${ }^{240} \mathrm{Pu}$ (c) nuclei, respectively. Experimental dots are obtained from the excited states spectrum of these nuclei (see Ref. [54]) by using the sample method, see text.

level density in Eq. (6). Mean value of oscillating $K(A)$ in Fig. 2 is about $8 \mathrm{MeV}$, as predicted in Ref. [9]. This is in accordance with the ETF (SkM* or KDE0v1) value, associated with the effective mass $m^{*}$. As shown in Ref. [19], the effect of the effective mass $m^{*}$ on the inverse level density parameter $K$ is strong, decreasing of $K$ by a factor of about 2, that leads approximately to mean values of the experimental data [13]. However, we should not expect that for the infinitely deep spherical square-well potential. The positions of the maxima [minima of $a$, i.e., of the single-particle level density $\delta g(\varepsilon)$ at $\varepsilon=\lambda$, related to magic nuclei in that potential] can not be correctly reproduced in such shell-correction calculations, because of neglecting the spin-orbit interaction. As shown in Refs. [44, 45], in order to reproduce the experimental value of $K$ for the magic nucleus ${ }^{240} \mathrm{Pu}$ in the semiclassical calculations, we should shift the curves $K(A)$ along the $A$ axis [through $k_{F} R$, Eq. (8)]. Therefore, we shifted the semiclassical curves in Fig. 2 with about $\Delta A=20$ along the particle number $A$ axis. This shift is of the order of the period $A_{\mathrm{sh}}$, Eq. (A.11), related to the distance $D_{\text {sh }}$, Eq. (A.10), between major shells near the Fermi surface in the particle number variable $A$. According to the POT estimations (see Appendix A and Ref. [43]) for the period $D_{\mathrm{sh}}$ and TF level density $g_{\mathrm{TF}} \sim A / \lambda$, one finds Eq. (A.11) for the particle number period of the major shell structure, $A_{\mathrm{sh}}$. For $A=100-200$, one obtains $A_{\mathrm{sh}} \approx 20-30$, which is of the order of the realistic period of the nuclear major shell structure; see also Ref. [48]. The position of a maximum over the particle number variable, as related to the value of $k_{F} R$, is the only one parameter needed to adjust the semiclassical solid curves in Fig. 2 and compare with the experimental data. This is similar to the discussions in Ref. [45] where the magic number for ${ }^{240} \mathrm{Pu}$ was obtained semiclassically by using a similar shift. Therefore, three minima of the level density shell corrections $\delta g$ for the major shell closures in the semiclassical calculations at $A \approx 45-150$ shown in Fig. 2 correspond to the maxima of the inverse level density parameter, $K \propto 1 / a$, experimentally obtained in Ref. [13]. In spite of very simple explicitly given analytical formulas (A.3) and (A.2) (Refs. [41-43]) for the level-density shell corrections in the spherical cavity, one obtains largely good agreement of the semiclassical approximation, which is almost identical to the quantum result of the shell-correction method for the same cavity, with the experimental data in this range of nuclear particle numbers $A$. The magnitudes of periods and amplitudes for the oscillations of $K(A)$ are basically in good agreement with experimental data. However, there is a discrepancy between experimental and theoretical results for $K(A)$ in the range of particle numbers $A \approx 150-240$. One of possible reasons is that different approximations for the statistical level density $\rho$ are used in fitting procedure 
to obtain the experimental prediction of $K$ with respect to those of the MMA approach (11). Experimental data for $K$ are in good agreement with those of neutron resonances, see Refs. [5, 19], which are dominating in the results of the calculations of Ref. [13]. The specific reason for the discrepancy might be that the level density parameter $a$ (or $K$ ) was obtained by three free parameter fit of these experimental data to the level density within the BSFG and constant temperature models. Another reason is that the pairing effects which, as well known, are strong in ${ }^{208} \mathrm{~Pb}$ should be accounted for such nuclei with a large pairing gap in the excited spectra, see Ref. [46].

In Fig. 3 we present results of the MMA and standard FG approximations to the statistical level density $\rho(E, A)$ (in logarithms) as functions of the excitation energy $U$ versus the experimental data. They are calculated employing the inverse level density parameter $K$ deduced from fits to experimental data on the excited energies in a low energystates (LES) range for several nuclei. The experimental data used for the statistical level density $\rho(E, A)$ are obtained from Ref. [54] for the excitation energies $U$ and spins with accounting for spin degeneracies by using the sample method [8, 46, 47]. In Fig. 3 we present the two opposite situations concerning the states distributions as functions of the excitation energy $U$. We show results for ${ }^{150} \mathrm{Sm}$ in Fig. 3(a) for the case of almost no states with extremely low excitation energies, similarly as for ${ }^{208} \mathrm{~Pb}$ where there is no such levels at all. Only a few levels in ${ }^{150} \mathrm{Sm}$ can be found at $U \lesssim 1 \mathrm{MeV}$, which yield entropies $S \lesssim 1$. For ${ }^{166} \mathrm{Ho}$ in Fig. 3(b), one finds the opposite situation when there is a lot of such LESs. An intermediate number of LESs is observed, e.g., in heavy ${ }^{240} \mathrm{Pu}$ (Fig. 3(c)). Thus, we present the results for nuclei, ${ }^{150} \mathrm{Sm}\left({ }^{166} \mathrm{Ho}\right)$, and ${ }^{240} \mathrm{Pu}$, from both sides of the desired particle number interval $A \approx 150-240$. In Fig. 3, we present the results of the MMA, Eqs. (12), (15) and (13), at the minimal control relative error-dispersion parameters of the least mean square fit:

$$
\sigma^{2}=\frac{\chi^{2}}{\aleph-1}, \quad \chi^{2}=\sum_{i} \frac{\left(y\left(U_{i}\right)-y_{i}^{\exp }\right)^{2}}{\left(\Delta y_{i}\right)^{2}}, \quad y=\ln \rho,
$$

where $\Delta y_{i} \sim 1 / \sqrt{N_{i}}, N_{i}$ is the number of the excited states in the ith sample, and $\boldsymbol{\aleph}$ is the sample number, $i=1,2, \ldots, \boldsymbol{\aleph}$. The number $N_{i}$ is large, according to the statistical condition of the sample method. The errors $\Delta y_{i}$ in statistical distributions of the excited states of the available experimental data over samples were evaluated as units of the theoretical versus experimental differences $y\left(U_{i}\right)-y_{i}^{\exp }$. We determine $\sigma$, Eq. (19), at the minimum of $\chi^{2}$ over the unique parameter, $K=K_{\min }$, having a definite physical meaning as the inverse level density parameter $K$ (see Fig. 2). Then, we may compare the $\sigma$ value of several different MMA approaches, which were found independently of the data, under certain statistical conditions mentioned above. For this aim we are interested in relative values of the $\sigma\left(K_{\min }\right)$ for different compared approximations rather than their absolute values. The MMA results for the minimal values of $\sigma$, Eq. (19), are shown in plots of Fig. 3 by red solid lines as best, among the MMA, approaches. As seen from these plots, they agree well with the experimental data. The results of our calculations are almost independent of the sample number, $\boldsymbol{\aleph}=5-7$, which plays the same role as an averaging parameter on the plateau condition, as in the Strutinsky averaging procedure [35].

In all panels of Fig. 3, one can see the divergence of the FG level density, see Eq. (18), near the zero excitation energy $U \rightarrow 0$, in contrast to the finite combinatoric MMA expressions (Refs. [2, 49, 50]) of Eq. (17) in this limit, see Eq. (11). We do not use empiric fitting parameters of the BSFG, in particular, spin cut-off parameter and those of the empiric constant temperature model [13]. As an advantage, as mentioned above, one has only one fitting parameter $K$ which has a certain physical meaning as the inverse level density parameter. The variations of $K$ are related, e.g., to those of the mean field parameters through Eq. (6), see Appendix A. All the MMA densities $\rho(E, A)$ do not depend on the cut-off spin factor and moment of inertia because of summation (integrations) over all spins (with accounting for the spin degeneracy factor) which could appear in the spectrum. The MMA results for ${ }^{150}$ Sm (MMA1, case (i)), ${ }^{166} \mathrm{Ho}$ (MMA2b, case (ii)), and ${ }^{240} \mathrm{Pu}$ (MMA2a, case (ii)) are compared with the results of the FG approximation and the experimental data in Fig. 3. Obviously, much better agreement with data is obtained for ${ }^{166}$ Ho (Fig. 3(b)) with a lot of states in the very LESs range. The MMA1 results are mainly agreed with experimental data for ${ }^{150} \mathrm{Sm}$ (Figure 3(a)), as well the FG approach, for which one has the opposite situation. For ${ }^{240} \mathrm{Pu}$ (Figure 3(c)), one obtains within the MMA (MMA2a) a better agreement with the experimental data at LESs than with the results of the FG formula (18). In any case, in the MMA, we removed the divergence of the statistical level density, $\rho(E, A)$, at small excitation energies $U$. Notice that the shell corrections effects measured by $\mathcal{E}_{\text {sh }}$, Eq. (10), increase going from (a) to (c) panels of Fig. 3, largely in agreement with the derivations of the MMA approaches.

In line with the results of Refs. [17, 21], the MMA results for $K$ can be significantly larger than the FG ones, and 
those obtained mainly for the neutron resonances. For instance, for very low excitation energy states in ${ }^{166} \mathrm{Ho}$, for the MMA2b approach one obtains $K \approx 17.6 \mathrm{MeV}(\sigma \approx 1.9)$ while for the FG approach one finds $K \approx 5.5 \mathrm{MeV}(\sigma \approx 11.1)$, cf. with the experimental data for neutron resonances, $K=9.7 \mathrm{MeV}$ in the same nucleus, see Fig. 2 and Ref. [13]. For MMA1, one finds $K$ of the same order of magnitude as that of the FG approach, in contrast to the MMA2b. The MMA1 $\left(K \approx 5.4 \mathrm{MeV}, \sigma \approx 12.1\right.$ for $\left.{ }^{166} \mathrm{Ho}\right)$ and $\mathrm{FG}$ values of $K$ are mostly close to those of neutron resonances in order of magnitude (Fig. 2). For the FG case it is obviously because they occur at large excitation energies $U$. Notice that in all our calculations of the statistical level density, $\rho(E, A)$, we did not use a popular assumption of small spins at large excitation energies which is valid for the neutron resonances. Large deformations, neutron-proton asymmetry and pairing correlations $[2,7,8,11,12,20,21]$ of the rare earth and actinide nuclei should be also taken into account $[46,47]$ to improve the comparison with experimental data.

\section{Conclusions}

We derived the statistical level density $\rho(S)$ as function of the entropy $S$ within the micro-macroscopic approximation using the mixed micro- and grand-canonical ensembles beyond the standard saddle point method of the Fermi gas model. This function can be applied for small and, relatively, large entropies $S$ or excitation energies $U$ of a nucleus. For a large entropy (excitation energy), one obtains the exponential asymptotes of the standard Fermi-gas model, however, with the significant inverse $1 / S$ power corrections. For small $S$ one finds the usual finite combinatoric expansion in powers of $S^{2} \propto U$. Functionally, the results of the MMA approach at linear approximation in $S^{2}$ expansion, and at small excitation energies, coincide with those of the empiric constant "temperature" model, but without using free fitting parameters. Thus, the MMA unifies the commonly accepted Fermi-gas approximation, for large entropies $S$, with the empiric constant temperature model for small entropies $S$, respectively, in line with the suggestions in Refs. [3, 20, 21]. The MMA results clearly manifest an advantage over the standard saddle point approaches, for the case of low excitation energies, because of no divergences of the MMA in the limit of small excitation energies, in contrast to the FG asymptote. Another advantage takes place for nuclei which have much more states in the very LES range. The values of the inverse level density parameter $\mathrm{K}$ are compared with experimental data for LESs which are significantly below neutron resonances in nuclear spectra of several nuclei. The MMA results with only one physical parameter in the least mean-square fits - inverse level density parameter $K$ - are usually the better the larger number of the extremely low energy states, certainly much better than for the FG model in this case. The MMA values of the inverse level density parameter $K$ for LESs can be significantly larger than those of the neutron resonances within the FG model. Major shell oscillations of the inverse level density parameter $K$ are compared with modern experimental data, basically for neutron resonances. We found qualitatively good agreement between semiclassical POT and quantum-mechanical results with experimental data on the inverse level density parameter $K$ for neutron resonances, after overall shift of all $K(A)$ curves between particle numbers $A \approx 45-150$ by only one parameter because of the spin-orbit interaction.

We have found significant shell effects in the MMA level density for the nuclear low excitation states (LES) range within the semiclassical periodic-orbit theory (POT). Therefore, a reasonable description of the LES experimental data for the statistical averaged level density obtained by the sample method within the MMA approach with the help of the semiclassical POT was achieved for several typical nuclei in the $A \approx 150-240$ range. We emphasize the importance of the shell effects in these calculations. We obtained values of $K$ that are significantly larger than those obtained for neutron resonances, due mainly to accounting for the shell effects. We show that the semiclassical periodic orbit theory is helpful in the LES range for analytical description of the level density and energy shell corrections. They are taken into account in the linear approximation up to small corrections due to the residual interaction beyond the mean field and extended Thomas-Fermi approximation within the shell-correction method, see Refs. [35, 36]. The main part of the interparticle interaction is described in terms of the extended Thomas-Fermi counterparts of the statistically averaged nuclear potential, and in particular, of the level density parameter. We obtained values of the inverse level density parameter $K$ for the LES range which are essentially different from those of neutron resonances. We found significantly larger $K$ values, than those for neutron resonances because of accounting for the shell effects.

As perspectives, the neutron-proton asymmetries, large nuclear angular momenta and deformations, as well as pairing correlations, will be taken into account in future work to significantly improve the comparison of the theoretical evaluations of the level density parameter with experimental data below the neutron resonances. Following the ideas of Refs. [6, 10, 14, 22, 53] and using the MMA results of Ref. [46], we are going to derive the spin-dependent level 
density for collective rotations of the axially symmetric deformed nuclei. We will study the enhancement factors of the level density due to their collective rotations and vibrations within the analytical MMA in the adiabatic approximation. Our approach can be applied also to the statistical analysis of the experimental data on collective nuclear states obtained in several nuclear reactions. The MMA approach with essential shell effects can be applied also for metallic clusters and quantum dots, and therefore, in solving several problems in nuclear astrophysics.

\section{Acknowledgement}

The authors gratefully acknowledge D. Bucurescu, R.K. Bhaduri, M. Brack, A.N. Gorbachenko, and V.A. Plujko for creative discussions. This work was supported in part by the budget program "Support for the development of priority areas of scientific researches", the project of the Academy of Sciences of Ukraine, Code 6541230, No 0122U000848. S. Shlomo is supported in part by the US Department of Energy under Grant no. DE-FG03-93ER40773.

\section{Appendix A. The semiclassical POT}

The level density shell corrections for a finite nucleon one-component system describing in the mean field approximation can be presented analytically within the periodic orbit theory (POT) in terms of the sum over classical periodic orbits (PO) [41, 43, 45],

$$
\delta g_{\mathrm{scl}}(\varepsilon)=\sum_{\mathrm{PO}} g_{\mathrm{PO}}(\varepsilon), \quad g_{\mathrm{PO}}(\varepsilon)=\mathcal{A}_{\mathrm{PO}}(\varepsilon) \cos \left[\frac{1}{\hbar} \mathcal{S}_{\mathrm{PO}}(\varepsilon)-\frac{\pi}{2} \mu_{\mathrm{PO}}-\phi_{0}\right] .
$$

Here $\mathcal{S}_{\mathrm{PO}}(\varepsilon)$ is the classical action along the PO in the potential well of radius, $R=r_{0} A^{1 / 3}\left(r_{0} \approx 1.1 \mathrm{fm}\right)$, $\mu_{\mathrm{PO}}$ is the so called Maslov index determined by the catastrophe points (turning and caustic points) along the PO, and $\phi_{0}$ is an additional shift of the phase coming from the dimension of the problem and degeneracy of the POs. The amplitude $\mathcal{A}_{\mathrm{PO}}(\varepsilon)$, as the action $\mathcal{S}_{\mathrm{PO}}(\varepsilon)$, are smooth functions of the single-particle energy $\varepsilon$, but, in addition, depend on the PO stability factors. The oscillating component, $\delta g(\varepsilon)$, Eq. (A.1), was approximated, with good accuracy, by the analytical POT trace formula [41-43] for the infinitely deep spherical square-well potential,

$$
\delta g_{\text {scl }}(k R)=\frac{2 m R^{2} d_{s}}{\hbar^{2}}\left[\sqrt{\frac{k R}{\pi}} \sum_{p, t}(-1)^{t} \sin \left(2 \varphi_{p t}\right) \sqrt{\frac{\sin \left(\varphi_{p t}\right)}{p}} \sin \left(k L_{p t}-3 p \frac{\pi}{2}+\frac{3 \pi}{4}\right)-\sum_{t} \frac{\sin (4 t k R)}{2 \pi t}\right],
$$

where $d_{s}$ is the spin (spin-isospin) degeneracy, $k=\sqrt{2 m \varepsilon / \hbar^{2}}$ is the wave number, $L_{p t}=2 p R \sin \left(\varphi_{p t}\right)$ is the PO length, $R$ is the radius of the spherical cavity, $\varphi_{p t}=\pi t / p$, and $p$ and $t$ are the numbers of turning points and rotations around the center of the PO (winding number) in the spherical cavity, respectively. The first and second terms in square brackets of Eq. (A.2) are the contributions of families of the planar orbits and diameters. The Gaussian local averaging of the level density shell correction, $\delta g_{\text {scl }}(\varepsilon)$, with a width $\Gamma$, over the single-particle energy spectrum $\varepsilon_{i}$ near the Fermi surface $\varepsilon_{F}$, for $\Gamma$ smaller than a distance between major shells, $\mathcal{D}_{\text {sh }}$, can be done analytically [41, 43, 45]. Similarly, one can average $\delta g_{\text {scl }}(k R)$ for cavity potentials over $k R$ variable with dimensionless $\gamma \approx \Gamma k_{F} R / 2 \lambda$, having

$$
\delta g_{\mathrm{scl}}^{(\gamma)}(k R) \cong \sum_{\mathrm{PO}} g_{\mathrm{PO}}(k R) \exp \left[-\left(\frac{\gamma L_{\mathrm{PO}}}{2 R}\right)^{2}\right]
$$

where $L_{\mathrm{PO}}$ is the length of the $\mathrm{PO}$ in a given cavity potential well, e.g. $L_{p t}$ for the $\mathrm{PO}(p, t)$ in the infinite square well potential.

Using the MMA approximation for calculations of the potential $\Omega$, one can express it in terms of the level density $g(\varepsilon)$, as

$$
\Omega \approx-\beta^{-1} \int_{0}^{\infty} \mathrm{d} \varepsilon g(\varepsilon) \ln \{1+\exp [\beta(\lambda-\varepsilon)]\}
$$


The level density $g(\varepsilon)$, within the Strutinsky shell-correction method [35] is a sum of the smooth, $\tilde{g}(\varepsilon)$, and oscillating shell, $\delta g(\varepsilon)$, components as

$$
g(\varepsilon) \cong \tilde{g}(\varepsilon)+\delta g(\varepsilon) .
$$

The oscillating part $\delta g(\varepsilon)$ is averaged over the single-particle energies near the Fermi energy using a small Gauss width, $\Gamma \propto \gamma$; see Eq. (A.3) for cavities in the POT and Ref. [35] for quantum-mechanical case of any potential well. Within the semiclassical POT $[41,43,44,46]$, the smooth and oscillating parts of the level density $g$ can be approximated, with good accuracy, by the ETF level density, $\tilde{g} \approx g_{\mathrm{ETF}}$, and the PO contribution, $\delta g \approx \delta g_{\text {scl }}$ [see Eq. (A.1)], respectively. Using the POT decomposition, Eq. (A.5), one finds from Eq. (A.4) that $\Omega=\tilde{\Omega}+\delta \Omega$, where $\tilde{\Omega} \approx \Omega_{\mathrm{ETF}}$ is the smooth ETF component $[24,33]$, given by

$$
\tilde{\Omega}=\tilde{E}-\lambda A-\frac{\pi^{2}}{6 \beta^{2}} \tilde{g}(\lambda) .
$$

The nuclear ETF energy component, $\tilde{E} \approx E_{\mathrm{ETF}}=\int_{0}^{\lambda} \mathrm{d} \varepsilon \varepsilon g_{\mathrm{ETF}}(\varepsilon)$, or the corresponding liquid-drop energy, is determined by a smooth chemical potential $\lambda \approx \tilde{\lambda}$ in the shell correction method. With the help of the POT [41, 43, 44, 46], one obtains $[33,46]$ for the oscillating (shell) component, $\delta \Omega$, see Eq. (A.4),

$$
\delta \Omega=-\beta^{-1} \int_{0}^{\infty} \mathrm{d} \varepsilon \delta g(\varepsilon) \ln \{1+\exp [\beta(\lambda-\varepsilon)]\} \cong \delta \Omega_{\mathrm{scl}}=\delta F_{\mathrm{scl}} .
$$

For the semiclassical free-energy shell correction, $\delta F_{\mathrm{scl}}$, or $\delta \Omega_{\mathrm{scl}}$, we incorporate the POT expression [33, 41]:

$$
\delta F_{\mathrm{scl}} \cong \sum_{\mathrm{PO}} F_{\mathrm{PO}}, \quad \text { with } \quad F_{\mathrm{PO}}=E_{\mathrm{PO}} \frac{x_{\mathrm{PO}}}{\sinh \left(x_{\mathrm{PO}}\right)}, \quad x_{\mathrm{PO}}=\frac{\pi t_{\mathrm{PO}}}{\hbar \beta},
$$

where $E_{\mathrm{PO}}$ is a $\mathrm{PO}$ component of the semiclassical shell correction energy, $\delta E_{\mathrm{scl}}$, given by

$$
E_{\mathrm{PO}}=\frac{\hbar^{2}}{\left(t_{\mathrm{PO}}\right)^{2}} g_{\mathrm{PO}}(\lambda), \quad \delta E_{\mathrm{scl}}=\sum_{\mathrm{PO}} E_{\mathrm{PO}} .
$$

Here $t_{\mathrm{PO}}=\mathrm{M} t_{\mathrm{PO}}^{\mathrm{M}=1}(\lambda)$ is the period of particle motion along a $\mathrm{PO}$ (taking into account its repetition, or period number $\mathrm{M})$, and $t_{\mathrm{PO}}^{\mathrm{M}=1}(\lambda)$ is the period of the particle motion along the primitive $(\mathrm{M}=1) \mathrm{PO}$ in the potential well with the radius $R=r_{0} A^{1 / 3}$. The period $t_{\mathrm{PO}}$ (and $t_{\mathrm{PO}}^{\mathrm{M}=1}$ ), and the partial oscillating level density component, $g_{\mathrm{PO}}$, are taken at the chemical potential, $\varepsilon=\lambda$; see also Eq. (A.1) for the semiclassical level-density shell correction (Refs. [41, 43]). The semiclassical expressions, Eqs. (A.6) and (A.7), are valid for a large relative action, $\mathcal{S}_{\mathrm{PO}} / \hbar \sim A^{1 / 3} \gg 1$. Then, expanding $x_{\mathrm{PO}} / \sinh \left(x_{\mathrm{PO}}\right)$, Eq. (A.8), in the shell correction $\delta \Omega$ [Eqs. (A.7) and (A.8)] in powers of $1 / \beta^{2}$ up to the quadratic terms, $\propto 1 / \beta^{2}$, for the excitation energies much smaller than the chemical potential, $U \ll \lambda$, see Ref. [46], one obtains Eq. (3). The chemical potential $\lambda$ ( or $\tilde{\lambda}$ ) is the solution of the conservation of particle numbers equation in Eq. (8). The POT shell component of the free energy, $\delta F_{\text {scl }}$, Eq. (A.8), is related in the nonthermal and nonrotational limit to the shell correction energy of a cold nucleus, $\delta E_{\mathrm{scl}}$; see Eq. (A.9) and Refs. [41, 43, 45]. Within the POT, $\delta E_{\mathrm{scl}}$ is determined, in turn, through Eq. (A.9) by the oscillating level density $\delta g_{\mathrm{scl}}(\varepsilon)$ at the chemical potential, $\varepsilon=\lambda$; see Eq. (A.1). The chemical potential $\lambda$ can be approximated by the Fermi energy $\varepsilon_{F}$ up to small excitation-energy corrections ( $T \ll \lambda$ for the saddle point value $T=1 / \beta^{*}$ if exists). It is determined by the particle-number conservation condition, Eq. (8), where $g(\varepsilon) \cong g_{\text {scl }}=g_{\text {ETF }}+\delta g_{\text {scl }}$ is the total POT level density. One now needs to solve the second equation of Eq. (8) to determine the chemical potential $\lambda$ as function of the particle numbers, $A$, since $\lambda$ is needed in Eq. (A.9) to obtain the semiclassical energy shell correction $\delta E_{\text {scl }}$.

For a major shell structure near the Fermi surface, $\varepsilon \approx \lambda$, the POT shell corrections, $\delta E_{\text {scl }}\left[\mathrm{Eq}\right.$. (A.9)] and $\delta g_{\text {scl }}(\lambda)$ [Eq. (A.1)] are, in fact, approximately proportional to each other. Indeed, the rapid convergence of the PO sum in Eq. (A.9) is guaranteed by the factor in front of the density component $g_{\mathrm{PO}}$, Eq. (A.1), a factor which is inversely proportional to the period time $t_{\mathrm{PO}}(\lambda)$ squared along the PO. Therefore, only POs with short periods which occupy a significant phase-space volume near the Fermi surface will contribute. These orbits are responsible for the major 
shell structure, that is related to a Gaussian averaging width, $\Gamma \approx \Gamma_{\mathrm{sh}}$, which is much larger than the distance between neighboring single-particle states but much smaller than the distance $D_{\text {sh }}$ between major shells near the Fermi surface. Eq. (A.3) for the averaged level density $\delta g(k R)$ as function of the wave-number variable $k R$ was derived under these conditions for averaging parameter $\gamma$, or dimensional Gauss width $\Gamma$ which are related to each other as described above. According to the POT [41, 43, 45], the distance between major shells, $D_{\mathrm{sh}}$, is determined by a mean period of the most short and degenerate POs, $\left\langle t_{\mathrm{PO}}\right\rangle$ (Refs. [41, 43]):

$$
\mathcal{D}_{\mathrm{sh}} \cong \frac{2 \pi \hbar}{\left\langle t_{\mathrm{PO}}\right\rangle} \approx \frac{\lambda}{A^{1 / 3}} .
$$

The period, $A_{\mathrm{sh}}$, of the oscillating part, $\delta K(A)$, of the inverse level density parameter, $\delta K \propto-A \delta g / \tilde{g}^{2}$ [see Eq. (6) for $a$ and Eq. (A.1) for $\delta g(\lambda)$ ] is defined approximately by the shell structure period $\mathcal{D}_{\text {sh }}$ of the level density $g$, Eq. (A.10), as function of the single-particle energy $\varepsilon$ near the Fermi surface, $\varepsilon \approx \lambda$. Using the relationship between the particle number $A$ and the chemical potential $\lambda$ through Eq. (8), one obtains for the particle number period of the shell structure, $A_{\text {sh: }}$ :

$$
A_{\mathrm{sh}} \approx \mathcal{D}_{\mathrm{sh}} \tilde{g} \sim A^{3 / 2}
$$

where the TF estimate, $\tilde{g} \approx g_{\mathrm{TF}} \sim A / \lambda$, was used.

Taking the factor in front of $g_{\mathrm{PO}}$ in Eq. (A.9) of the shell correction energy $\delta E_{\mathrm{scl}}$ off the sum over the POs for the semiclassical energy-shell correction [43-45], one arrives at

$$
\delta E_{\mathrm{scl}} \approx\left(\frac{D_{\mathrm{sh}}}{2 \pi}\right)^{2} \delta g_{\mathrm{scl}}(\lambda)
$$

Differentiating Eq. (A.1) for $g_{\mathrm{PO}}(\lambda)$ over $\lambda$ in Eq. (A.9), one can keep only the dominating terms coming from differentiation of the cosine of the action phase argument, $S / \hbar \sim A^{1 / 3}$. Finally, one finds the relationships which are useful in our derivations (Sec. 2):

$$
\frac{\partial^{2} \delta E_{\mathrm{PO}}}{\partial \lambda^{2}} \approx-\delta g_{\mathrm{PO}}, \quad \frac{\partial^{2} g_{\mathrm{scl}}}{\partial \lambda^{2}} \approx \sum_{\mathrm{PO}} \frac{\partial^{2} \delta g_{\mathrm{PO}}}{\partial \lambda^{2}} \approx-\left(\frac{2 \pi}{D_{\mathrm{sh}}}\right)^{2} \delta g_{\mathrm{scl}}(\lambda) .
$$

\section{References}

[1] H. Bethe, Phys. Rev. 50 (1936) 332

[2] T. Ericson, Adv. in Phys. 9 (1960) 425.

[3] A. Gilbert, A. G. W. Cameron Canadian J. of Phys. 43 (1965) 1446

[4] A. Bohr, B. R. Mottelson, Nuclear structure, vol. I (Benjamin, New York, 1969).

[5] V. S. Stavinsky, Sov. J. Part. Nucl. 3 (1972) 417.

[6] S. Bjørnholm, A. Bohr, and B. R. Mottelson, Role of symmetry of the nuclear shape in rotational contributions to nuclear level densities: In Proc. of Symposium on the physics and chemistry of fission, Rochester, USA, 1973, vol. 1 (Int. At. Energy Agency, Vienna, 1974), pp. 367-373.

[7] A. V. Ignatyuk, Statistical properties of excited atomic nuclei (Energoatomizadat, Moscow, 1983 (Russian)).

[8] Yu. V. Sokolov, Level density of atomic nuclei (Energoatomizadat, Moscow, 1990 (Russian)).

[9] S. Shlomo, Nucl. Phys. A539 (1992) 17.

[10] A. R. Junghans, M. de Jong, H.-G. Clerc, A. V. Ignatyuk, G. A. Kudyaev, K.-H. Schmidt, Nucl. Phys. A 629, 635 (1998)

[11] Y. Alhassid, G. F. Bertsch, S. Liu, H. Nakada, Phys. Rev. C 84 (2000) 4313.

[12] Y. Alhassid, G.F. Bertsch, L. Fang, Phys. Rev. C 68 (2003) 044322.

[13] T. von Egidy, D. Bucurescu, Phys. Rev. C 78 (2008) 051301(R);

T. von Egidy, D. Bucurescu, Phys. Rev. C 80 (2009) 054310.

[14] S. M. Grimes, Phys. Rev. C 88 (2013) 024613.

[15] Y. Alhassid, M. Bonett-Matiz, S. Liu, H. Nakada, Phys. Rev. C 92 (2015) 024307.

[16] Y. Alhassid, G. F. Bertsch, C. N. Gilbreth, H. Nakada, Phys. Rev. C 93 (2016) 044320.

[17] V. Zelevinsky, R. Senkov, Phys. Rev. C 93 (2016) 064304.

[18] S. Karampagia, V. Zelevinsky, Phys. Rev. C 94 (2016) 014321.

[19] V.M. Kolomietz, A.I. Sanzhur, S. Shlomo, Phys. Rev. C 97 (2018) 064302.

[20] V. Zelevinsky, S. Karampagia, Eur. Phys. J. Web Conf. 194 (2018) 01001.

[21] V. Zelevinsky, M. Horoi, Prog. Part. Nucl. Phys. 105 (2019) 180.

[22] S. M. Grimes, T. N. Massey, A. V. Voinov, Phys. Rev. C 99 (2019) 064331. 
[23] S. Karampagia, V. Zelevinsky, Int. J. Mod. Phys. E 29 (2020) 2030005.

[24] V. M. Kolomietz, S. Shlomo, Mean Field Theory (World Scientific, 2020).

[25] V. Zelevinsky, B. A. Brown, N. Frazier, M. Horoi, Phys. Rep. 276 (1996) 85.

[26] J. M. G. Gomez, K. Kar, V. K. B. Kota, R. Molina, A. A. Relano, J. Retamosa, Phys. Rep. 499 (2011) 103.

[27] A. G. Magner, A. I. Levon, S. V. Radionov, Eur. Phys. J. A 54 (2018) 214.

[28] A. I. Levon, D. Bucurescu, C. Costache, T. Faestermann, R. Hertenberger, A. Ionescu, R. Lica, A. G. Magner, C. Mihai, R. Mihai, C. R. Nita, S. Pascu, K. P. Shevchenko, A. A. Shevchuk, A. Turturica, H.-F. Wirth, Phys. Rev. C 102 (2020) 014308.

[29] W. E. Ormand, Phys. Rev. C 56 (1997) R1678.

[30] F. Borgonovi, F. M. Israilev, L. F. Santos, V. G. Zelevinsky, Phys. Rep. 626 (2016) 1.

[31] C. E. Porter, Statistical Theories of Spectra: Fluctuactions, (Academic Press, 1965).

[32] M. L. Mehta, Random Matrix Ensembles in Quantum Physics, 3rd ed. (Elsevier, Amsterdam, 2004).

[33] V. M. Kolomietz, A. G. Magner, V. M. Strutinsky, Sov. J. Nucl. Phys. 29 (1979) 758.

[34] V. M. Strutinsky, Nucl. Phys. A 95 (1967) 420;

V. M. Strutinsky, Nucl. Phys. 122 (1968) 1.

[35] M. Brack, L. Damgaard, A. S. Jensen, A. C. Pauli, V. M. Strutinsky, C. Y. Wong, Rev. Mod. Phys. 44 (1972) 320.

[36] G. G. Bunatian, V. M. Kolomietz, V. M. Strutinsky, Nucl. Phys. A 188 (1972) 225.

[37] A. B. Migdal, The Finite Fermi-System Theory and Properties of Atomic Nuclei (Intersience, New York, 1967; Ibid. Nauka, Moscow, 1983).

[38] V. A. Khodel, E. E. Saperstein, Phys. Rep. 5 (1982) 183.

[39] W. D. Myers, W. J. Swiatecki, Ann. Phys. (N.Y.) 55 (1969) 395; W. D. Myers, W. J. Swiatecki, Ann. Phys. (N.Y.) 84 (1974) 186.

[40] M. Brack, C. Guet, H.-B. Håkansson, Phys. Rep. 123 (1985) 275.

[41] M. Brack, R. K. Bhaduri, Semiclassical Physics. Frontiers in Physics, No. 96, 2nd ed. (Westview Press, Boulder, CO, 2003).

[42] R. Balian, C. Bloch, Ann. Phys. 69 (1972) 76.

[43] V. M. Strutinsky, A. G. Magner, Sov. J. Part. Nucl. 7 (1976) 138.

[44] V. M. Strutinsky, A. G. Magner, S. R. Ofengenden, T. Døssing, Z. Phys. A 283 (1977) 269.

[45] A. G. Magner, A. S. Yatsyshyn, K. Arita, M. Brack, Phys. At. Nucl. 74 (2011) 1445.

[46] A. G. Magner, A. I. Sanzhur, S. N. Fedotkin, A. I. Levon, S. Shlomo, Phys. Rev. C, 104 (2021) 044319.

[47] A. G. Magner, A. I. Sanzhur, S. N. Fedotkin, A. I. Levon, S. Shlomo, Int. J. Mod. Phys. E 30 (2021) 2150092.

[48] P. Moeller, A.J. Sierk, T. Ichikawa, H. Sagawa, Atomic Data and Nuclear Data Tables, Atom. Data $\mathcal{G}$ Nucl. Data Tables 109-110, 1-204 (2016); http://www.elsevier.com/locate/adt.

[49] V. M. Strutinsky, On the nuclear level density in case of an energy gap: In Proc. of Int. Conf. on Nucl. Phys. (Paris, 1958), pp. 617-622.

[50] A. V. Ignatyuk, Yu. V. Sokolov, Yadern. Fiz. 16 (1972) 217; Preprint FEI-327, (FEI, Obninsk, 1972), pp. 1-19.

[51] W. Dilg, W. Shantl, M. Uhl, Nucl. Phys. A 217 (1973) 269.

[52] B. K. Agrawal, S. Shlomo, V. K. Au, Phys. Rev. C 72 (2005) 014310

[53] A. Bohr, B. R. Mottelson, Nuclear Structure, vol. 2 (Benjamin, New York, 1975).

[54] National Nuclear Data Center On-Line Data Service for the ENSDF (Evaluated Nuclear Structure Data File) database, http://www.nndc.bnl.gov/ensdf. 